\title{
Fiebre y sus implicaciones en el diagnóstico del paciente neurológico, revisión narrativa de la literatura
}

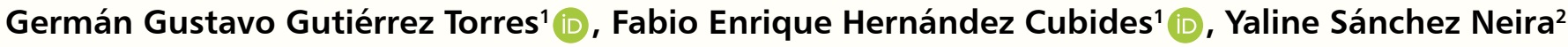

\section{RESUMEN}

Introducción: La fiebre se considera uno de los primeros síntomas registrados en pacientes hospitalizados y se ha convertido en un importante marcador de enfermedad. La presente revisión buscó recopilar evidencia en torno a la fiebre y su importancia en los diferentes escenarios clínicos que se presentan en el paciente neurológico, ya que en los últimos años se ha logrado comprender mejor el papel que desempeña el sistema nervioso central dentro de la termorregulación.

Metodología: Búsqueda de la literatura en bases de datos como Pubmed, OVID, Epistemonikos, SciELO y Google Scholar, durante los años 2009 al 2019 en español e inglés, sobre fiebre e implicaciones en pacientes neurológicos, usando palabras clave como fiebre, sensibilidad, especificidad, likelihood ratio, enfermedades del sistema nervioso, neurología, encefalitis, neuritis y traumatismo del sistema nervioso.

Resultados: Se encontraron 374 artículos, de los cuales 48 cumplieron con los criterios de inclusión. Se incluyeron libros de texto, como metaanálisis, revisiones sistemáticas, revisiones scoping, artículos de revisión y estudios observacionales. Se analizaron y subdividieron en 8 apartados con temáticas en común para su estudio.

Conclusiones: En etapas tempranas de eventos cerebro-vasculares isquémicos o hemorrágicos la fiebre se comporta como una respuesta sistémica secundaria al daño de base, y es crucial corregirla tempranamente. Al mismo tiempo, en traumatismos del sistema nervioso central, la fiebre en las primeras 72 horas se identifica como un marcador de mal pronóstico.

Palabras clave: neurología; fiebre signos vitales; enfermedad del sistema nervioso; diagnóstico.

${ }^{1}$ Clínica de Especialistas, Sogamoso, Colombia.

${ }^{2}$ Universidad de Boyacá, Tunja, Colombia.

Autora de correspondencia: Yaline Sánchez Neira. Correo electrónico: ysanchez@uniboyaca.edu.co

Citar este artículo así:

Gutiérrez Torres GG, Hernández Cubides FE, Sánchez Neira Y. Fiebre y sus implicaciones en el diagnóstico del paciente neurológico: revisión narrativa de la literatura. Rev Investig Salud Univ Boyacá. 2021;8(1):170-185. https://doi.org/10.24267/23897325.568 


\begin{abstract}
Introduction: Fever is considered one of the first symptoms registered in hospitalized patients, becoming an important marker of disease. The present review sought to collect evidence on fever and its importance in the different clinical scenarios that occur in neurological patients, since in recent years it has been possible to better understand the role of the central nervous system within the thermoregulation.
\end{abstract}

Methodology: A literature search was carried out in databases such as Pubmed, OVID, EpistemonikOs, SciELO and Google Scholar during the years 2009 to 2019 in Spanish and English languages, on fever and implications in neurological patients, using keywords such as fever, sensitivity, specificity, likelihood ratio, diseases of the nervous system, neurology, encephalitis, neuritis and trauma of the nervous system.

Results: A total of 374 articles were found, of which 48 articles met the inclusion criteria, for the construction of this review article. Textbooks, as well as meta-analyzes, systematic reviews, scoping reviews, and review articles were included. They were analyzed and subdivided into 8 sections with common themes for their study.

Conclusions: In early stages of ischemic or hemorrhagic CVD, fever behaves as a systemic response secondary to the underlying injury, and it is crucial to correct it early. At the same time, in CNS trauma, fever in the first 72 hours is identified as a poor prognostic marker.

Keywords: neurology; fever; vital signs; nervous system disease; diagnosis. 


\title{
Febre e suas implicações no diagnostico do paciente neurológico, uma revisão narrativa da literatura
}

\begin{abstract}
RESUMO
Introdução: A febre é considerada um dos primeiros sintomas registrados em pacientes hospitalizados e tornou-se um importante marcador de doença. A presente revisão procurou reunir evidencias em torno da febre e sua importância nos diferentes panoramas clínicos no paciente neurológico, uma vez que nos últimos anos foi possível compreender melhor o papel do sistema nervoso central no processo de termo regulação.
\end{abstract}

Metodologia: Foi realizada uma pesquisa de literatura em bases de dados como Pubmed, OVID, Epistemonikos, SciELO e Google Acadêmico, durante os anos 2009 a 2019 em espanhol e inglês, sobre febre e suas implicações em pacientes neurológicos, utilizando palavras-chave como febre, sensibilidade, especificidade, likelihood ratio, doenças do sistema nervoso, neurologia, encefalite, neurite e trauma do sistema nervoso.

Resultados: Foi encontrado um total de 374 artigos, dos quais 48 abrangiam os critérios de inclusão. Foram incluídos livros de texto, como meta-análises, revisões sistemáticas, revisões Scoping, artigos de revisão e estudos observacionais. Foram analisadas e subdivididas em 8 secções com assuntos comuns para seu estudo.

Conclusões: Em fases iniciais de eventos cerebrovasculares isquêmicos ou hemorrágicos, a febre se comporta como uma resposta sistêmica secundária ao dano de base, e é crucial corrigi-la precocemente. Enquanto, em traumatismos do sistema nervoso central, a febre nas primeiras 72 horas é identificada como um marcador de mau prognóstico.

Palavras-chave: neurologia; febre; sinais vitais; doença do sistema nervoso; diagnóstico. 


\section{INTRODUCCIÓN}

La práctica médica, y por ende su enseñanza, está ligada a avances tecnológicos disponibles que contribuyen a mejorar el diagnóstico y tratamiento médico. Ello reposiciona la clínica con el uso apropiado de la tecnología en la práctica médica (1). Así mismo, el estudio de síntomas y signos hallados en los pacientes durante las observaciones clínicas es la base fundamental y reproducible en otras patologías similares $(2,3)$, lo cual contribuye no solo con el diagnóstico de las enfermedades, sino con la enseñanza tradicional de la medicina. $Y$ aunque en algunos casos se han limitado las capacidades del médico y creado incertidumbre sobre los signos físicos encontrados, la oportuna atención a las necesidades del paciente e importancia de evaluar las alteraciones encontradas en el examen clínico y su relevancia orientan hacia un diagnóstico certero (1-4).

En 2009, Simel y Rennie (5) llevaron a cabo una serie de investigaciones que exponen conceptos básicos sobre medicina basada en la evidencia. En ellas se toman distintas patologías y se describe el contexto clínico, la sensibilidad y especificidad de los signos y síntomas más frecuentes. Dentro de ellos se destacan la fiebre, la inflamación, la sudoración, el vómito, la tos, el sarpullido, la tensión arterial alta, la frecuencia cardiaca y respiratoria, entre otros. De igual forma, Villegas y Villegas (6) abordaron las constantes vitales y su interpretación clínica, situando una descripción del término, las bases de su fisiología, factores que afectan los niveles normales y los instrumentos para su medición. En efecto, de esta forma, se visualiza la creciente necesidad de llevar a cabo investigaciones que mejoren la práctica clínica, la enseñanza médica $y$, por consiguiente, ofrezcan mejores resultados sobre los pacientes $(7,8)$.

La fiebre fue uno de los primeros síntomas en ser registrados rutinariamente en pacientes hospitalizados, al considerarse un importante marcador de enfermedad. Así, a través de los últimos años, se ha logrado comprender mejor el papel que desempeña el sistema nervioso central (SNC) con el control neurohormonal dentro de la termorregulación ejercida en el hipotálamo anterior $(4,9)$, ya que ante episodios de temperaturas elevadas, se presentan alteraciones en los pacientes en la función del SNC, que se caracterizan por confusiones, somnolencia o alucinaciones. $Y$ si estos valores de temperaturas siguen en aumento, los daños son irreversibles: desde un estado de coma hasta la muerte. Por lo anterior, la presente revisión buscó recopilar evidencia publicada en la última década (2009-2019) con la fiebre y su representación en la neurología como un aporte a la constante actualización de los conocimientos médicos y su enseñanza en las escuelas de medicina. 


\section{METODOLOGÍA}

Se llevó a cabo una revisión narrativa de la información existente sobre fiebre y sus implicaciones en pacientes neurológicos durante los años 2009 al 2019. Como criterios de inclusión se tomaron las publicaciones científicas desde el 2009 hasta el 2019, que estuvieran disponibles en español e inglés, incluyendo trabajos que hubieran involucrado población adulta. Dentro de los criterios de exclusión se tuvieron en cuenta las cartas al editor, editoriales, población infantil y embarazo, por considerarse grupos con distintos factores de riesgo y cambios fisiológicos subyacentes a su condición (9).

Se consultaron las bases de datos Pubmed, OVID, Epistemonikos, SciELO y Google Scholar, a partir de la búsqueda combinada de literatura que incluyera las palabras clave: fiebre, sensibilidad, especificidad, likelihood ratio, enfermedades del sistema nervioso, neurología, encefalitis, neuritis y traumatismo del sistema nervioso. En la búsqueda inicial se obtuvieron 374 artículos, de los cuales se eliminaron 326 , que no cumplían con los criterios de selección. Finalmente, se seleccionaron 48 artículos, de los cuales se extrajo la información relevante para la construcción del presente artículo de revisión (figura 1).

\section{RESULTADOS}

Para el abordaje de la fiebre y la enfermedad neurológica, en la presente revisión narrativa de la literatura se encontraron las siguientes categorías: fiebre como signo clínico, evento cerebrovascular isquémico, fiebre y hemorragia intracerebral, trauma del SNC y fiebre, neoplasias y patologías autoinmunes, infecciones febriles y paciente neurológico y fiebre en el paciente crítico.

\section{Fiebre como signo clínico}

También conocida como pirexia, representa una respuesta adaptativa al estrés fisiológico, como parte de una respuesta inflamatoria sistémica, que genera efectos benéficos o nefastos $(10,11)$. Fisiológicamente, la regulación de la temperatura corporal está dada por el hipotálamo en el núcleo preóptico, aunque también se ha evidenciado que participan los centros focales del puente; de ahí que lesiones a estos centros termorreguladores resulten en hipertermia (12). El aumento de la temperatura ocurre cuando el termoefector incrementa su actividad, y como resultado se obtienen valores por encima de $37,8^{\circ} \mathrm{C}$, de tal forma que consensos mundiales consideran la fiebre superior a $38^{\circ} \mathrm{C}$ y otros la toman como un valor superior a $38,3^{\circ} \mathrm{C}$, este último particularmente en pacientes que se encuentran en unidades de cuidado intensivo $(11,13)$. 
Figura 1. Proceso de selección de la revisión sobre fiebre y representación neurológica

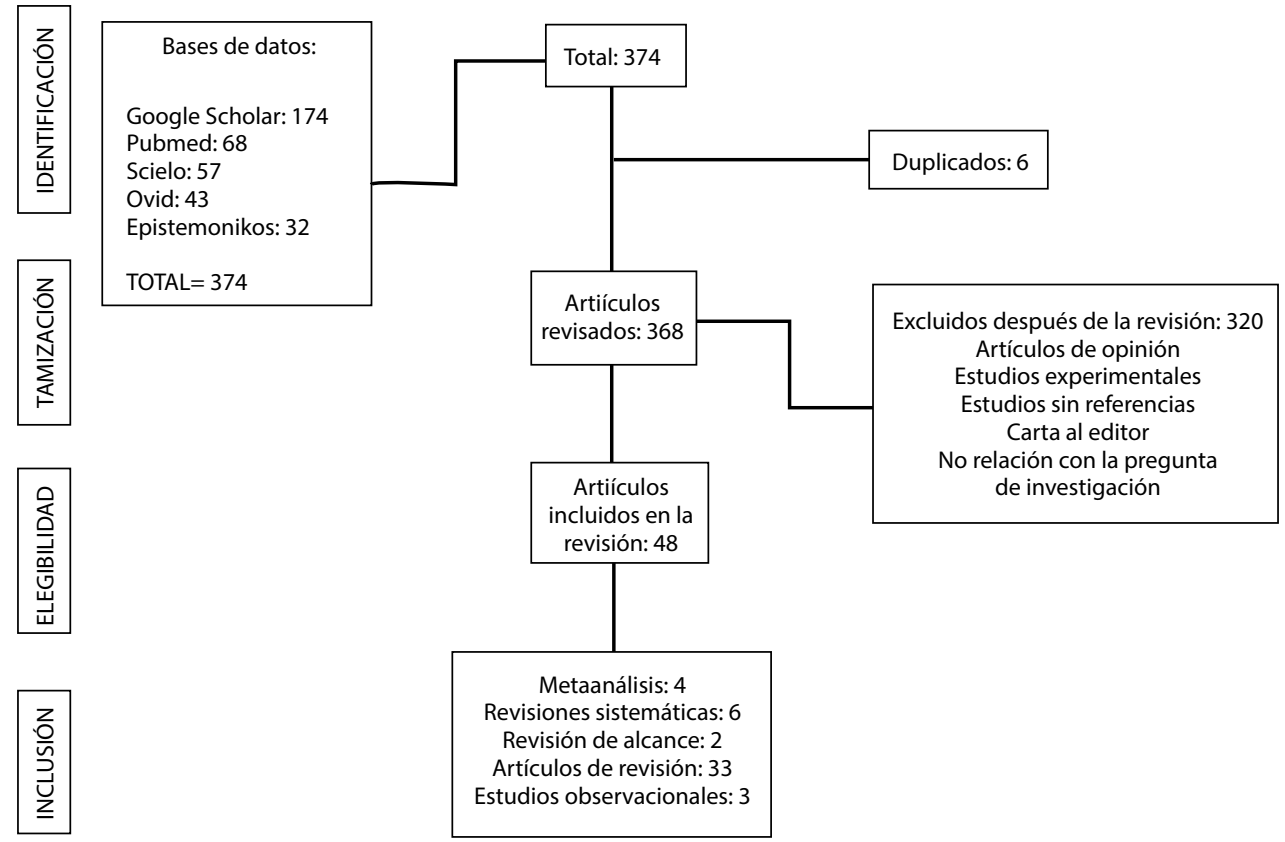

La fiebre se genera como una respuesta adaptativa provocada, en la gran mayoría de las ocasiones, por microrganismos, aun cuando también participan respuestas hormonales, autoinmunes y a veces medicamentosas, que generan complejos cambios neuroquímicos y metabólicos que en principio buscan controlar la agresión, brindando un efecto protector $(11,12)$. Algunas condiciones, como los tumores cerebrales, la hemorragia subaracnoidea (HSA), la hemorragia intraventricular y el vasoespasmo se han asociado con el desarrollo de fiebre de origen central (14-16).
Se debe considerar la fiebre como un signo relevante que alerta, en muchos casos, sobre la necesidad de realizar intervenciones oportunas y apropiadas (17)relatively little is known about the symptoms associated with febrile states. The purpose of this study was to assess the validity, reliability and feasibility of the Fever Assessment Tool (FAST. En algunos otros, se deben sopesar los posibles efectos adversos de la administración tanto de antipiréticos como de antibióticos, al pensar que se trata de una infección en un paciente con patología neurológica $(10,18)$. 


\section{Fiebre en evento cerebrovascular isquémico}

La elevación de la temperatura en el inicio de un evento cerebrovascular isquémico corresponde a la respuesta sistémica causada por la isquemia cerebral, mediada por un incremento del tono simpático y mecanismos inflamatorios constituidos principalmente por citocinas proinflamatorias; mientras que al ocurrir tardíamente, corresponde pensar en complicaciones asociadas con daño neurológico, como infecciones periféricas o trombosis venosa profunda $(12,19)$.

La identificación y control oportuno de causas subyacentes a la fiebre en enfermedad cerebrovascular puede garantizar la disminución de la morbilidad en dichos pacientes, pues se han reportado casos en los cuales la pirexia sería un signo que amerita un análisis profundo, por la presencia de patologías asociadas, por ejemplo, neumonías aspirativas o colecistitis aguda $(20,21)$. De igual forma, hay que considerar la administración de medicamentos pues, según estudios recientes, la presencia de hipertermia posterior a la administración de un trombolítico como el rt-PA estaría asociado con un peor pronóstico funcional para el paciente (19). Así mismo, Mantero et al. (22), en el 2018, encontraron que la fiebre se comportaría como un factor predictor de deterioro neurológico temprano y el más importante que se debería corregir de manera pronta.

\section{Fiebre y hemorragia intracerebral}

La hemorragia intracerebral es un evento complejo con alta incidencia de morbilidad y mortalidad (23). La fiebre puede considerarse parte de la sintomatología del proceso hemorrágico si no hay infecciones asociadas o si esta se presenta dentro de las primeras 72 horas de inicio del evento $(24,25)$ and it has been associated with the occurrence of delayed cerebral ischemia and worse outcomes in previous studies. Frequently, fever is not the result of bacterial infections, and distinction between infection-related fever and fever secondary to brain injury (also referred as central fever. Sin embargo, la hipertermia ha demostrado ser un factor de mal pronóstico que aumenta la morbilidad y la mortalidad de los pacientes, en especial cuando se produce más tempranamente o cuando perdura tiempos prolongados, porque ello causa un daño de carácter secundario $(15,23,24,26-28)$.

Existen reportes de episodios febriles en el contexto de la hemorragia intracerebral, en particular con HSA, en los cuales a la fiebre se le atribuye un origen central y su incidencia varía entre el $70 \%$ y el $80 \%$ de los casos $(12,25,26,28)$. El origen de estos episodios de hipertermia neurogénica está dado por un daño en el hipotálamo, al aumentar la vasoconstricción con la disminución del flujo sanguíneo junto con un incremento en la producción de prostaglandinas y citocinas proinflamatorias, que 
ocasionan un daño hemotóxico con posterior muerte axonal $(12,15,23)$.

En este contexto, la fiebre se vincula con la posterior presencia de isquemia, y su persistencia puede desencadenar peores resultados que acentúan el edema cerebral, la presión intracraneal y, por consiguiente, disminuye el grado de conciencia $(25,28)$. Dado que se han asociado peores resultados, en cuanto a mortalidad, estancia hospitalaria y secuelas, surge la necesidad de una adecuada termorregulación. Estudios como el de Suehiro et al. (25) han establecido una posible relación entre el control temprano de la fiebre y un mejor pronóstico en pacientes con HSA que fueron llevados a intervenciones quirúrgicas.

De igual forma, es preciso recordar que se pueden hacer presentes otras causas de fiebre en el paciente con hemorragia intracerebral, dadas por infecciones, las cuales, en su gran mayoría, aparecerán luego de los tres primeros días del inicio del sangrado (26). Las infecciones con la fiebre como su primer síntoma se relacionan con mayor estancia hospitalaria (24). Y aunque todavía no es totalmente claro qué beneficio representan las medidas de control de temperatura, sí es importante tener en cuenta sus principales orígenes y evaluar juiciosamente la necesidad del uso tanto de antipiréticos como de antibióticos, con el fin de disminuir las interacciones medicamentosas y la resistencia bacteriana (25-27).

\section{Trauma del sistema nervioso central y fiebre}

En todo el mundo, el trauma severo sobre el SNC implica una gran mortalidad y morbilidad en los pacientes menores de 40 años, independientemente de si la afección es cerebral o en la médula espinal (29), pues cuando hay trauma del SNC y fiebre durante las primeras 72 horas del evento, ello se ha asociado con mayores desenlaces fatales $(29,30)$. Estos resultados desfavorables están relacionados con la ruptura de la barrera hematoencefálica, producción de radicales libres con toxicidad celular y alteraciones hidroelectrolíticas que, al combinarse con la pirexia, promueven su efecto nocivo en el SNC y el estado general del paciente (29).

En el caso del trauma raquimedular, la hipertermia temprana puede ser un predictor de disfunción autónoma; mientras que al presentarse en estadios más tardíos, se asocia con infecciones de origen pulmonar, urinario o dermatológico, en ocasiones promovidas por el uso de dispositivos médicos, como la ventilación mecánica, los catéteres centrales o las sondas vesicales (30-32).

La prolongación de la fiebre en estos pacientes se ha vinculado con mayor daño neurológico $y$, consecuentemente, con peor pronóstico, puesto que se les afecta la función inmunitaria, que inhibe la apoptosis de las células inmunitarias y amplía la 
respuesta de citocinas proinflamatorias estimuladas no solo por infecciones, sino también por sangrado $(32,33)$. En un estudio desarrollado por Shi et al. (34) se demostró que en los pacientes existe un riesgo cuatro veces mayor de presentar delírium cuando requirieron intervenciones neuroquirúrgicas y presentaron fiebre durante su recuperación posoperatoria. Ello indica, al mismo tiempo, una pobre recuperación del daño físico causado.

\section{Neoplasias y patologías autoinmunes}

Reconocida a lo largo del tiempo por el personal sanitario, la fiebre se ha contemplado como uno de los principales signos físicos de inflamación y respuesta sistémica; no obstante, se reconoce que las patologías autoinmunes y los tumores pueden causar diversas manifestaciones de tipo neurológico que, en principio, requieren estudios diferenciales detallados $(35,36)$. Es importante tener en cuenta que estos síndromes febriles, en su mayoría, son de características recurrentes, en los cuales se asocian síntomas neurológicos, como cefalea, afasia, confusión, cambios en el estado de ánimo, alucinaciones, disminución neurosensorial de la audición, alteraciones sensitivas y motoras, entre otros (36-38).

En el momento del abordaje del paciente, hay que considerar que existen propuestas como el EUROFEVER, que buscan optimizar la atención del paciente con cuadros febriles recurrentes, no asociados a causa infecciosa y sin estudios previos que pueden corresponder a patologías autoinmunes (37). De igual forma, se debe considerar la necesidad de atención urgente a pacientes con fiebre y focalización neurológica aguda, dado que pueden presentarse como manifestaciones inespecíficas de neoplasias o una combinación de condiciones previas desconocidas con complicaciones agudas $(38,39)$.

\section{Infecciones febriles y paciente neurológico}

Tradicionalmente, se ha considerado la fiebre como un marcador agudo de infección; sin embargo, recientes estudios han demostrado que, aun en presencia de infección, se puede retrasar su aparición y que puede ser un error confiar en su presencia como signo orientador del diagnóstico (40-42). Estudios recientes como los de Zhou et al. (43), Yusuf et al. (44), que analizaron la presencia la tríada clásica de infección del SNC -caracterizada por fiebre, cefalea y déficit neurológico-, demostraron que el signo más prevalente fue la fiebre, con una representación del $55 \%$, y que la tríada completa solo se encontró en fases tardías de la enfermedad, lo cual demuestra una baja sensibilidad (8\%), pero un alta especificidad (99\%) para el diagnóstico de neuroinfección.

En paralelo, hay combinaciones, como el dolor de espalda y fiebre, que presentan una fuerte 
asociación con el diagnóstico de infecciones de la médula espinal; sin embargo, es de especial cuidado su estudio, pues no siempre están presentes los dos síntomas y, de igual forma, se puede retrasar el diagnóstico y tratamiento oportuno $(45,46)$.

En el proceso diagnóstico es importante tener en cuenta patologías o reacciones que pueden ser confusoras y requieran nuestra total atención pues, como se ha relatado, hay signos clínicos que no suelen ser tan precisos e implican un mayor análisis a la hora de dar un dictamen médico acertado $(42,47)$. Por ejemplo, en un país de carácter tropical como Colombia se hace necesario mencionar las enfermedades trasmitidas por vectores que nos pueden ampliar el rango de enfermedades febriles, aunadas a otras manifestaciones neurológicas (como el dengue, el cólera, la fiebre amarilla $\mathrm{o}$, incluso, otras infecciones ascendentes, como la encefalitis del Nilo Occidental, entre otras), las cuales son capaces de producir cuadros de meningitis y encefalitis severos $\mathrm{y}$ llevar el paciente a la muerte $(40,48,49)$.

\section{Fiebre en el paciente crítico}

Como ya se ha documentado, las reacciones febriles en los paciente críticos son de mal pronóstico neurológico $(10,14,18)$. Y aquí es importante mencionar a los pacientes que se encuentran en cuidados posparto cardiaco, en quienes se ha documentado que, probablemente producto de la hipoxia e isquemia cerebral secundaria, el presentar fiebre se asocia con peores resultados neurológicos y con menores tasas de supervivencias, por lo cual la hipotermia se ha recomendado como una medida terapéutica apropiada para aquellos pacientes en cuidados posparo cardiaco (50).

\section{CONCLUSIONES}

La fiebre se genera como una respuesta adaptativa sistémica al daño que recibe el cuerpo por parte de diferentes agresores. En el paciente neurológico es de suma importancia reconocer su origen y darle el tratamiento apropiado. En etapas tempranas de enfermedad cerebrovascular, isquémica o hemorrágica, la fiebre se comporta como una respuesta sistémica secundaria al daño de base, por lo que es crucial corregirla lo más rápido posible. Al mismo tiempo, en traumatismo del SNC, la fiebre en las primeras 72 horas se identifica como un marcador de mal pronóstico. A futuro son necesarios estudios que evalúen la precisión de los signos clínicos y su respectivo aporte en el proceso diagnóstico de los pacientes con patología neurológica, dado que la semiología es un pilar fundamental para obtener óptimos resultados en favor de la salud del paciente. 


\section{LIMITACIONES}

En el presente estudio de revisión de la literatura se presentaron algunas limitaciones en la variable tiempo, al relacionarla con fiebre, signos y síntomas prevalentes en enfermedades, ya que al inicio del periodo, que fue incluido en la búsqueda de literatura en el texto de los artículos, no eran claros y específicos los síntomas descritos. Por otro lado, no se cuenta con el acceso a textos completos en algunas publicaciones de interés.

\section{CONFLICTO DE INTERESES}

Los autores declaran no tener ningún conflicto de intereses.

\section{REFERENCIAS}

1. Japp AG, Robertson C, Wright RJ, Reed $M$, Robson A. Macleod's clinical diagnosis. 2. ${ }^{\mathrm{a}}$ ed. Edinburgh: Elsevier Health Sciences; 2018.

2. Serra Valdés MÁ, Viera García M. Consideraciones sobre la enseñanza de la semiología, la propedéutica y el proceso diagnóstico en la práctica clínica. Rev Cuba Educ Med Super [internet]. 2014;28(1):163-74. Disponible en: http://scielo.sld.cu/scielo.php?script=sci_artte xt\&pid =S0864-21412014000100017
3. Argente $\mathrm{H}$, Álvarez M E. Semiología médica: fisiopatología, semiotecnia y propedéutica. Enseñanza-aprendizaje centrada en la persona. 2.a ed. Buenos Aires: Editorial Médica Panamericana; 2013.

4. McGee S, editor. Evidence-based physical diagnosis. Philadelphia: Elsevier; 2018. https://doi.org/10.1016/B978-1-4160-2898-7. X5001-1

5. Simel DL, Rennie D. The rational clinical examination: evidence-based clinical diagnosis. JAMA-McGraw-Hill; 2009.

6. Villegas J, Villegas OA. Semiología de los signos vitales: una mirada novedosa a un problema vigente. Arch Med. 2012;12(2):221-40. https:// doi.org/10.30554/archmed.12.2.10.2012

7. Jinich H, Lifshitz A, García J HR. Síntomas y signos cardinales de las enfermedades. 7. ${ }^{\mathrm{a}}$ ed. Vol. 7. Ciudad de México: Manual Moderno; 2017.

8. Mccolgan P, Mckeown PP, Selai C, Doherty-Allan $\mathrm{R}$, Mccarron MO. Educational interventions in neurology: a comprehensive systematic review. Eur J Neurol. 2013;20(7):1006-16. https://doi.org/10.1111/ene.12144 
9. Harden LM, Kent S, Pittman QJ, Roth J. Fever and sickness behavior: friend or foe? Brain Behav Immun. 2015;50(July):322-33. https:// doi.org/10.1016/j.bbi.2015.07.012

10. Niven DJ, Stelfox HT, Laupland KB. Antipyretic therapy in febrile critically ill adults: a systematic review and meta-analysis. J Crit Care. 2013;28(3):303-10. https://doi. org/10.1016/j.jcrc.2012.09.009

11. Romero González JP, Carrillo-Esper R, MezaMárquez JM, Sosa-García JO. Actualidades en el tratamiento de la fiebre en el paciente con sepsis y choque séptico: controversias y recomendaciones basadas en evidencia. Med Int Méx [internet]. 2017;33(1):99108. Disponible en: http://www.scielo. org. $\mathrm{mx} / \mathrm{scielo}$.php? script $=$ sci_arttext $\&$ pid $=$ S0186-48662017000100099

12. Badjatia N. Hyperthermia and fever control in brain injury. Crit Care Med. 2009;37(7):S250-7. https://doi.org/10.1097/ CCM.0b013e3181aa5e8d

13. Rhodes A, Evans LE, Alhazzani W, Levy $M M$, Antonelli $M$, Ferrer $R$, et al. Surviving sepsis campaign: international guidelines for management of sepsis and septic shock. Intens Care Med. 2017;43:304-77. https://doi. org/10.1007/s00134-017-4683-6
14. Hocker SE, Tian L, Li G, Steckelberg JM, Mandrekar JN, Rabinstein AA. Indicators of centralfeverintheneurologicintensivecareunit. JAMA Neurol. 2013;70(12):1499-504. https:// doi.org/10.1001/jamaneurol.2013.4354

15. Gillow SJ, Ouyang B, Lee VH, John S. Factors associated with fever in intracerebral hemorrhage. J Stroke Cerebrovasc Dis. 2017;26(6):1204-8. https://doi.org/10.1016/j. jstrokecerebrovasdis.2017.01.007

16. Saadat S. Predictors of prolonged hospitalization in patients with fever. Cureus. 2016;8(9). http://doi:10.7759/cureus.789

17. Ames NJ, Powers JH, Ranucci A, Gartrell K, Yang $L$, VanRaden $M$, et al. A systematic approach for studying the signs and symptoms of fever in adult patients: the fever assessment tool (FAST). Health Qual Life Outcomes. 2017;15(1):1-11. https://doi.org/10.1186/ s12955-017-0644-6

18. Sánchez-Díaz JS, Martínez-Rodríguez EA, Peniche-Moguel G, Huanca-Pacaje JM, López-Guzmán C, Calyeca-Sánchez MV. Fiebre en el paciente críticamente enfermo: ¿tratar o no tratar? Med Int Méx [internet]. 2017;33(1):48-60. Disponible en: http://www. scielo.org.mx/scielo.php? script $=$ sci_arttext\&p id $=$ S0186-48662017000100048 
19. Ueno $T$, Nishijima $H$, Hikichi $H$, Haga $R$, Arai $A$, Suzuki $C$, et al. Association of survival and hyperthermia after rt-PA for ischemic stroke. Acta Neurol Scand. 2018;138(6):574-8. https://doi.org/10.1111/ane.13011

20. Schwarz M, Coccetti A, Murdoch A. The impact of aspiration pneumonia and nasogastric feeding on clinical outcomes in stroke patients: a retrospective cohort study. J Clin Nurs. 2017;27(1-2):235-41. https://doi. org/10.1111/jocn.13922

21. Kuroi $Y$, Imazato D, Yamazaki K, Kasuya $H$. Acute cholecystitis in patients with stroke. Neurol India. 2019;67(2):439-41. https://doi. org/10.4103/0028-3886.258055

22. Mantero V, Scaccabarozzi C, Botto E, Giussani G, Aliprandi A, Lunghi $A$, et al. Outcome in lacunar stroke: a cohort study. Acta Neurol Scand. 2018;138(4):320-6. https://doi. org/10.1111/ane.12961

23. Honig A, Michael S, Eliahou R, Leker RR. Central fever in patients with spontaneous intracerebral hemorrhage: predicting factors and impact on outcome. BMC Neurol. 2015;15(1):1-7. https://doi.org/10.1186/ s12883-015-0258-8
24. Rocha Ferreira da Silva I, Rodriguez de Freitas G. Early predictors of fever in patients with aneurysmal subarachnoid hemorrhage. J Stroke Cerebrovasc Dis. 2016;25(12):2886-90. https://doi.org/10.1016/j.jstrokecerebrovasd is.2016.07.051

25. Suehiro $E$, Sadahiro $H$, Goto $H$, Oku T, Oka $F$, Fujiyama $Y$, et al. Importance of early postoperative body temperature management for treatment of subarachnoid hemorrhage. J Stroke Cerebrovasc Dis. 2016;25(6):1482-8. https://doi.org/10.1016/j.jstrokecerebrovasd is. 2016.01 .053

26. Magee CA, Thompson Bastin ML, Graves K, Burgess D, Nestor M, Lamm JR, et al. Fever burden in patients with subarachnoid hemorrhage and the increased use of antibiotics. J Stroke Cerebrovasc Dis. 2019;28(11):1-9. https://doi.org/10.1016/j.jstr okecerebrovasdis.2019.104313

27. Lord AS, Karinja S, Lantigua H, Carpenter A, Schmidt JM, Claassen J, et al. Therapeutic temperature modulation for fever after intracerebral hemorrhage. Neurocrit Care. 2014;21(2):200-6. https://doi.org/10.1007/ s12028-013-9948-5

28. Scaravilli V, Tinchero G, Citerio G. Fever management in SAH. Neurocrit Care. 
2011;15(2):287-94. https://doi.org/10.1007/ s12028-011-9588-6

29. Bao L, Chen D, Ding L, Ling W, Xu F. Fever burden is an independent predictor for prognosis of traumatic brain injury. PLoS One. 2014;9(3):3-7. https://doi.org/10.1371/ journal.pone.0090956

30. Hinson $H$, Schreiber M, Laurie A, Baguley I, Bourdette D, S, Ling G. Early fever as a predictor of paroxysmal sympathetic hyperactivity in traumatic brain injury. Physiol Behav. 2019;176(3):139-48. https://doi.org/10.1097/ HTR.0000000000000271

31. Trbovich M, Li C, Lee S. Does the CDC definition of fever accurately predict inflammation and infection in persons with $\mathrm{SCl}$ ? Top Spinal Cord Inj Rehabil. 2016;22(4):260-8. https://doi. org/10.1310/sci2016-00049

32. Wang Z, Shen M, Qiao M, Zhang H, Tang Z. Clinical factors and incidence of prolonged fever in neurosurgical patients. J Clin Nurs. 2017;26(3-4):411-7. https://doi.org/10.1111/ jocn.13409

33. Seguin $P$, Roquilly $A$, Mimoz $O$, Maguet $P L$, Asehnoune K, Biederman S, et al. Risk factors and outcomes for prolonged versus brief fever: a prospective cohort study. Crit Care.
2012;16(4):R150. https://doi.org/10.1186/ cc11465

34. Shi C, Yang C, Gao R, Yuan W. Risk factors for delirium after spinal surgery: a meta-analysis. World Neurosurg. 2015;84:1466-72. https:// doi.org/10.1016/j.wneu.2015.05.057

35. Ames NJ, Peng C, Powers JH, Kline Leidy $\mathrm{N}$, Miller-Davis $\mathrm{C}$, Rosenberg $\mathrm{A}$, et al. Beyond intuition: patient fever symptom experience. J Pain Symptom Manage. 2013;46(6):807-16. https://doi.org/10.1016/j. jpainsymman.2013.02.012

36. Zeft AS, Spalding SJ. Autoinflammatory syndromes: fever is not always a sign of infection. Cleve Clin J Med. 2012;79(8):56981. https://doi.org/10.3949/ccjm.79a.11184

37. Federici S, Sormani MP, Ozen S, Lachmann HJ, Amaryan G, Woo P, et al. Evidence-based provisional clinical classification criteria for autoinflammatory periodic fevers. Ann Rheum Dis. 2015;74(5):799-805. https://doi. org/10.1136/annrheumdis-2014-206580

38. Gago-Veiga AB, Díaz de Terán J, GonzálezGarcía N, González-Oria C, GonzálezQuintanilla A, Minguez-Olaondo A, et al. Cómo y cuándo derivar un paciente con cefalea secundaria y otros tipos de dolores 
craneofaciales desde urgencias y atención primaria: recomendaciones del Grupo de Estudio de Cefaleas de la Sociedad Española de Neurología. Soc Española Neurol. 2017;35(5):323-31. https://doi.org/10.1016/j. nrl.2017.08.002

39. Cosacov R, Spadaro E. Accidente cerebrovascular como manifestación inicial de arteritis de Takayasu y revisión de casos en Argentina. Neurol Argentina. 2011;3(4):237-9. https://doi.org/10.1016/j.neuarg.2011.06.006

40. Rhee C, Kharod GA, Schaad N, Furukawa NW, Vora NM, Blaney DD, et al. Global knowledge gaps in acute febrile illness etiologic investigations: A scoping review. PLoS Negl Trop Dis. 2019;13(11):1-16. https:// doi.org/10.1371/journal.pntd.0007792

41. Rabinstein AA. Herpes virus encephalitis in adults: current knowledge and old myths. Neurol Clin. 2017;35(4):695-705. https://doi. org/10.1016/j.ncl.2017.06.006

42. Waghdhare S, Kalantri A, Joshi R, Kalantri S. Accuracy of physical signs for detecting meningitis: A hospital-based diagnostic accuracy study. Clin Neurol Neurosurg. 2010;112(9):752-7. https://doi.org/10.1016/j. clineuro.2010.06.003
43. Zhou W, Shao X, Jiang X. A clinical report of two cases of cryptogenic brain abscess and a relevant literature review. Front Neurosci. 2019;13(jan):1-7. https://doi.org/10.3389/ fnins.2018.01054

44. Yusuf M, Finucane L, Selfe J. Red flags for the early detection of spinal infection in back pain patients. BMC Musculoskelet Disord. 2019;20(1):1-10. https://doi.org/10.1186/ s12891-019-2949-6

45. Davis WT, April MD, Mehta S, Long B, Shroyer $S$. High risk clinical characteristics for pyogenic spinal infection in acute neck or back pain: prospective cohort study. Am J Emerg Med. 2019;38(3):491-6. https://doi.org/10.1016/j. ajem.2019.05.025

46. Smids C, Kouijzer IJE, Vos FJ, Sprong T, Hosman AJF, de Rooy JWJ, et al. A comparison of the diagnostic value of MRI and 18F-FDG-PET/ $\mathrm{CT}$ in suspected spondylodiscitis. Infection. 2017;45(1):41-9. https://doi.org/10.1007/ s15010-016-0914-y

47. Arando $M$, Fernández-Naval C, Mota-Foix M, Álvarez A, Armegol P, Barberá MJ, et al. The Jarisch-Herxheimer reaction in syphilis: could molecular typing help to understand it better? J Eur Acad Dermatology Venereol. 2018;32(10):1791-5. https://doi.org/10.1111/ jdv.15078 
48. Ralapanawa DMPUK, Kularatne SM, Jayalath WATA. Guillain-Barre syndrome following dengue fever and literature review. BMC Res Notes. 2015;8(1). https://doi.org/10.1186/ s13104-015-1672-0

49. Farrar F. West Nile virus: an infectious viral agent to the central nervous system. Crit Care Nurs Clin North Am. 2013;25(2):191-203. https://doi.org/10.1016/j.ccell.2013.02.005

50. Grossestreuer AV, Gaieski DF, Donnino MW, Wiebe DJ, Abella BS. Magnitude of temperature elevation is associated with neurologic and survival outcomes in resuscitated cardiac arrest patients with postrewarming pyrexia. J Crit Care. 2017;38:78-83. https://doi. org/10.1016/j.jcrc.2016.11.003

Esta obra está bajo una licencia de Creative Commons Reconocimiento-NoComercial 4.0 Internacional 